'Departamento de Salud Pública y Epidemiología. Facultad de Medicina. Universidad de los Andes. Santiago, Chile.

${ }^{2}$ Department of Population Health, London School of Hygiene and Tropical Medicine. Londres, Reino Unido.

${ }^{3}$ Facultad de Educación. Pontificia Universidad Católica de Chile. Santiago, Chile.

${ }^{4}$ Escuela de Enfermería, Campus San Felipe. Universidad de Valparaíso. San Felipe, Chile.

${ }^{5}$ School of Nursing, Chang Gung University of Science and Technology, Taoyuan, Taiwan. aPsicólogo, PhD.

${ }^{b}$ Enfermera Universitaria, MSc.

'Enfermera Universitaria, PhD.

Fuente de apoyo financiero: Este estudio cuenta con el apoyo en su financiamiento por la Comisión Nacional de Ciencia y Tecnología (CONICYT) a través del concurso FONIS Elige Vivir Sano: FONIS EVS 1310100: Influencias parentales en los estilos de vida saludable de adolescentes chilenos: un estudio longitudinal.

Recibido el 26 de noviembre de 2015, aceptado el 6 de junio de 2016.

Correspondencia a: Dr. Jorge Gaete London School of Hygiene \& Tropical Medicine Keppel Street London, WC1E 7HT jorge.gaete@Ishtm.ac.uk

\section{Influencia de las conductas promotoras de salud de los padres en la de sus hijos adolescentes}

\author{
JORGE GAETE ${ }^{1,2}$, CRISTIAN A. ROJAS-BARAHONA ${ }^{3, a}$, \\ ESTERBINA OLIVARES ${ }^{4, b}$, MEI-YEN CHEN ${ }^{5, c}$
}

\section{The influence of parental healthy behaviors on healthy lifestyles of early adolescents}

Background: Family behavior models may influence health promoting conducts among adolescents. Aim: To determine the association between health promoting behaviors among parents and healthy behaviors of early adolescents. Material and Methods: Analysis of the baseline assessment of a longitudinal study of early adolescents in the city of San Felipe, Region of Valparaiso, Chile. Parents and their teenage children, attending $5^{\text {th }}$ to $7^{\text {th }}$ grade, from ten municipal schools, participated in this study. Self-reported questionnaires were used to assess healthy lifestyles, answered separately by parents and their children. Univariable and multivariable ordinal logistic regression analyses with complete data were carried out, using the students' health promoting behaviors as dependent variables and the same behaviors among parents as the main predictors, controlling for other personal and family variables. Results: We contacted 1,035 parents and 682 consented to participate along with 560 students. The mean age of adolescents was $11.5 \pm 1.2$ years ( $49 \%$ females) The mean age of parents was $39.8 \pm 8.8$ years and $90 \%$ were women. The parental behaviors associated with teenage health promoting behaviors were eating vegetables (odds ratio $(O R)=$ $1.22, p<0.05)$, having breakfast $(\mathrm{OR}=1.27, p<0.05)$, do stretching exercises every day $(O R=1.19, p<0.05)$ and take some time for relaxation $(O R=1.24$, $p<0.05)$. Conclusions: These results show an association between healthy behaviors among parents and these behaviors among their adolescent offspring.

(Rev Med Chile 2016; 144: 870-878)

Key words: Adolescent; Family; Health Behavior; Health Promotion; Life Style.
1 1 desarrollo de enfermedades crónicas como obesidad, diabetes mellitus e hipertensión arterial en adultos está relacionado con los estilos de vida que se van adquiriendo en etapas tempranas de la vida ${ }^{1}$, especialmente durante la adolescencia temprana (10-14 años) $)^{2}$. Las conductas saludables o promotoras de salud son aquellas que, por un lado, favorecen la salud o estado de bienestar del individuo (como el ejercicio físico o una alimentación balanceada $)^{3}$, y por otro, redu- cen los riesgos en salud (como el evitar el consumo de sustancias de abuso).

Los determinantes de conductas son múltiples $\mathrm{y}$ actúan a distintos niveles ${ }^{3-8}$. A nivel personal, la salud mental del adolescente juega un rol y la relación parece ir en ambas direcciones. ${ }^{9}$. Por ejemplo, un estudio mostró que la presencia de síntomas emocionales redujo la probabilidad de tener conductas saludables ${ }^{10}$, mientras otro, mostró que practicar actividad física redujo el riesgo 
de estar triste ${ }^{11}$. Por otro lado, la percepción de salud general predice la morbi-mortalidad y uso de servicios ${ }^{12}$, esto parece instalarse temprano en la vida, y depende de varios factores ${ }^{13}$, como disconformidad corporal ${ }^{14}$, menor actividad física y rendimiento escolar ${ }^{15,16}$. Asimismo, la disconformidad con la apariencia física y peso parecen disminuir la motivación para hacer ejercicios y adoptar conductas saludables ${ }^{16,17}$. Otros factores que parecen influir son el tiempo que los adolescentes pasan viendo televisión o jugando en el computador o juegos de consola ${ }^{18}$, reflejando el sedentarismo que amenaza hoy a los adolescentes.

En relación a factores familiares, una revisión mostró que los modelos conductuales presentados en la familia, como el tipo y cantidad de la ingesta alimenticia, determinarían la conducta de los niños y adolescentes ${ }^{19}$. Por otro lado, ciertas dinámicas familiares, especialmente en relación a la comunicación con los padres, tienen una clara influencia en el desarrollo adolescente y conductas de salud ${ }^{20,21}$.

Pocos estudios en el mundo han investigado la influencia directa de las conductas saludables de los padres o apoderados sobre estas mismas conductas en sus hijos adolescentes, y los estudios que lo han hecho han explorado alguna conducta, como es la alimentación ${ }^{19}$ y no a través del estudio de varias conductas a la vez. Para el conocimiento de los autores, no hay estudios en Latinoamérica que hayan investigado esta asociación.

El objetivo de esta investigación es determinar la asociación entre conductas saludables reportadas por los padres/apoderados y conductas similares reportadas por estudiantes de $5^{\circ}$ a $7^{\circ}$ Básicos, evaluado por distintos factores personales $\mathrm{y}$ familiares.

\section{Material y Método}

\section{Participantes}

La muestra fue por conveniencia, invitando a participar a todos los establecimientos educacionales municipales urbanos de la comuna de San Felipe (Región de Valparaíso, Chile), que impartieran educación básica general $(\mathrm{n}=10$ colegios). Se contactó a 1.035 padres/apoderados de alumnos de $5^{\circ}$ a $7^{\circ}$ Básicos, de los cuales 682 consintieron; y de estos, 560 alumnos asintieron participar (10 no asintieron y 112 alumnos estuvieron ausentes el día de la evaluación).

\section{Recolección de datos}

Los consentimientos y cuestionarios de auto-reporte de los padres/apoderados fueron enviados y recibidos durante los meses de agosto y septiembre de 2014 para cuatro establecimientos educacionales y en marzo de 2015 para los otros seis establecimientos. El desfase en la evaluación basal de estos últimos seis colegios se debió a un paro de actividades académicas a fines del año 2014.

Los alumnos fueron evaluados en octubre y noviembre de 2014 para los primeros cuatro colegios, y en abril y mayo de 2015 para los otros seis colegios. Dos ayudantes de investigación por curso solicitaron firmar asentimiento y completar el cuestionario en forma colectiva en fecha, horario y lugar proporcionado por las autoridades del establecimiento educacional.

\section{Instrumentos}

\section{Conductas promotoras de salud}

Para el estudio específico de las conductas promotoras de salud, se seleccionaron de los instrumentos Escala de Promoción de Salud del Adolescente (AHP) ${ }^{22}$ y del Cuestionario de Estilos de Vida II (HPLP2) ${ }^{23,24}$ para adultos, sólo aquellos ítems que fueran exactamente comparables, es decir, que el contenido de la afirmación fuera el mismo o evaluara el mismo constructo. Así, fueron incluidas 20 afirmaciones (Tabla 1). Para cada afirmación, a mayor puntaje, mayor frecuencia de esa conducta saludable. Ambos instrumentos están inspirados en el modelo de promoción de salud de Nola Pender ${ }^{3}$.

\section{Otras variables de alumnos}

Se preguntó por: edad, sexo, curso, percepción del estado de salud general, conformidad con peso y apariencia física, número de horas diarias viendo televisión y sentido psicológico de membresía escolar.

La salud mental fue evaluada a través del Cuestionario de Fortalezas y Dificultades (SDQ) ${ }^{25}$ en su versión para adolescentes. Consta de 25 afirmaciones sobre emociones y conductas que los alumnos pueden tener en los últimos 6 meses. Es decir, se refiere a un estado transitorio de psicopatología, y no necesariamente a uno crónico. Consta de 5 sub-escalas (cuatro sobre problemáticas y una de fortalezas), de 5 ítems cada una: i) Síntomas emo- 
Tabla 1. Afirmaciones presentes en ambas escalas

\begin{tabular}{|c|c|c|}
\hline & $\begin{array}{c}\text { Escala } \\
\text { Adolescentes } \\
\text { (nº pregunta) }\end{array}$ & $\begin{array}{c}\text { Escala } \\
\text { Padres } \\
\text { (n॰ pregunta) }\end{array}$ \\
\hline \multicolumn{3}{|l|}{ Nutrición } \\
\hline Me preocupo de comer alimentos que contienen poca grasa & 13 & 12 \\
\hline Incluyo fibra en mi dieta (por ejemplo, frutas y verduras) & 14 & $\begin{array}{l}30 \text { (frutas) } \\
36 \text { (vegetales) }\end{array}$ \\
\hline Tomo desayuno todos los días & 17 & 60 \\
\hline \multicolumn{3}{|l|}{ Apoyo social } \\
\hline Me preocupo por lo demás & 19 & 35 \\
\hline Hablo de mis preocupaciones con otras personas & 20 & 11 \\
\hline Trato de tener buenos amigos & 23 & 23 \\
\hline Hablo de mis problemas con otros & 24 & 61 \\
\hline \multicolumn{3}{|l|}{ Responsabilidad en salud } \\
\hline Acostumbro leer las etiquetas de los productos alimenticios & 25 & 54 \\
\hline $\begin{array}{l}\text { Hablo acerca de cuestiones de salud con mis profesores o padres o profesionales de } \\
\text { la salud }\end{array}$ & 27 & 49 \\
\hline Observo con detención mi cuerpo al menos 1 vez al mes & 28 & 43 \\
\hline Leo o veo información sobre la salud (por ejemplo, en Internet o en televisión) & 31 & 19 \\
\hline \multicolumn{3}{|l|}{ Apreciación de la vida } \\
\hline Trato de sentirme feliz y contento(a) & 34 & 34 \\
\hline Intento comprender mis fortalezas y debilidades y las acepto & 36 & 27 \\
\hline Hago el intento de saber qué es importante para mí & 38 & 52 \\
\hline Hago el esfuerzo por creer que mi vida tiene sentido & 40 & 22 \\
\hline \multicolumn{3}{|l|}{ Actividad física } \\
\hline Hago ejercicios para estirarme diariamente (ejercicios de elongación) & 41 & 38 \\
\hline Hago ejercicio vigorosamente por al menos 30 min, 3 veces a la semana & 42 & 20 \\
\hline \multicolumn{3}{|l|}{ Manejo del estrés } \\
\hline Trato de tener tiempo para relajarme & 46 & 21 \\
\hline Duermo $6-8 \mathrm{~h}$ cada noche & 49 & 15 \\
\hline Planifico mis horarios y fijo mis prioridades & 50 & 45 \\
\hline
\end{tabular}

Nota: La numeración de los ítems sigue el orden como fueron presentados en los cuestionarios a los alumnos y a los padres/ apoderados.

cionales; ii) Problemas de conducta; iii) Problemas de hiperactividad y desatención; iv) Problemas con pares; y v) Conducta prosocial. Aún no se encuentran disponibles en Chile normas para la población general, ni puntajes de cortes para discriminar entre casos y no casos. Sin embargo, hay evidencia que indica que el SDQ es una buena medida dimensional de psicopatología, por lo que es adecuado su uso como un puntaje continuo, indicando que a mayor puntaje en las sub-escala de problemáticas, mayor es la probabilidad de psicopatología ${ }^{25}$. Por otro lado, a mayor puntaje en la sub-escala de fortalezas o de habilidades sociales, menor probabilidad de presentar problemas de convivencia que requieran atención.
Otras variables de padres/apoderados

Se preguntó sobre edad, sexo, estado civil y nivel educacional.

Se evaluó el estado de salud mental general a través del Cuestionario de Salud General de 12 ítems (GHQ-12), validado en Chile ${ }^{26}$. Contiene 12 afirmaciones y el puntaje total se calcula sumando todos los ítems: a mayor puntaje, peor es la percepción del estado de salud mental general.

El funcionamiento familiar se evaluó a través del cuestionario Escala Breve de Evaluación Familiar Faces 20 ESP, la cual contiene 20 afirmaciones que pueden ser contestadas en una escala de $1=$ Nunca o casi nunca, a $5=$ Casi siempre. Este cuestionario evalúa la adaptabilidad y co- 
hesión familiar, y está validado en Chile ${ }^{27}$. Para este estudio se utilizó la escala total, y el puntaje se calcula sumando las respuestas a cada ítem. A mayor puntaje, mejor percepción del funcionamiento familiar.

\section{Análisis estadístico}

La descripción de la muestra consideró el uso de medias y desviación estándar para las variables continuas, y porcentajes e intervalos de confianza a $95 \%$ para el caso de variables categoriales.

El estudio de asociación se realizó a través de modelos de regresión logística ordinal por la naturaleza de las variables. Se consideró como variables dependientes a cada uno de los 20 ítems del cuestionario de estudiantes que tuviera un ítem de similar contenido en el cuestionario de sus padres. Para facilitar la interpretación de los resultados, se transformaron los coeficientes de regresión en odd ratios (OR).

En una primera etapa se realizaron modelos no ajustados, para cada variable dependiente. Luego se construyeron modelos finales ajustados sólo para las variables promotoras de salud de los alumnos que estuvieron asociadas la conducta correspondiente de los padres con un nivel de significancia de $\mathrm{p}<0,05$. Para el caso de las otras variables independientes, de los estudiantes y de apoderados, sólo pasaron a estos modelos finales, las variables que también tuvieran una asociación con un nivel de significancia de $\mathrm{p}<0,05$.

Todos los análisis se realizaron en el paquete estadístico STATA 12.1.

\section{Consideraciones éticas}

Estudio aprobado por el Comité de Bioética de la Facultad de Medicina de la Universidad de los Andes (18 de junio de 2014) y está de acuerdo con la Declaración de Helsinki ${ }^{28}$. Todos los participantes dieron consentimiento (apoderados) y asentimiento (alumnos) por escrito.

\section{Resultados}

\section{Características de la muestra}

Un total de 560 parejas apoderado/alumno proporcionaron información para este estudio. El $49,1 \%$ de los estudiantes fueron mujeres. La edad media fue de 11,5 años ( $\mathrm{DE}=1,2)$. El 60\% de los estudiantes vivía con ambos padres. Las principales problemáticas en salud mental de esta población fueron la presencia de síntomas emocionales (Media $=4,5, \mathrm{DE}=2,3$ ) y síntomas de desatención e hiperactividad (Media $=4,5$, $\mathrm{DE}=2,3)($ Tabla 2).

Entre los padres/apoderados, $89,6 \%$ de ellos fueron mujeres. La edad media fue de 39,8 $(\mathrm{DE}=8,8)$. En relación al parentesco, $87,7 \%$ fueron sus madres, $6,6 \%$ sus padres y $3,4 \%$ sus abuelas. El 39,1\% era dueña de casa y 35,8\% refirió trabajar tiempo completo fuera de la casa. El $39,8 \%$ de los padres/apoderados no completó sus estudios de enseñanza media.

\section{Asociación entre conductas saludables en los apoderados y estudiantes}

A nivel univariable, de las 20 conductas pareadas entre adolescentes y padres, siete de ellas estuvieron asociadas significativamente, representando a los dominios de nutrición, actividad física y manejo del estrés de las sub-escalas originales de la AHP (Tabla 3). Por ejemplo, a mayor frecuencia del consumo de vegetales por parte del apoderado, mayor era la frecuencia del consumo de fibras (sean frutas o vegetales) entre los adolescentes; o también, a mayor frecuencia de destinar de tiempo para relajarse, mayor probabilidad que los hijos reporten lo mismo.

A nivel multivariable, es decir, ajustando por otras variables individuales y familiares, las conductas de comer vegetales, tomar desayuno, realizar ejercicios de estiramiento diariamente y destinar tiempo para relajarse entre los apoderados, se asociaron a estas mismas conductas en los adolescentes.

\section{Otras variables asociadas a conductas promotoras de salud de adolescentes}

Considerando sólo las variables ingresadas en los modelos multivariables, y excluyendo lo ya mencionado en relación a las conductas promotoras de salud de los apoderados, encontramos además que: 1) comer frecuentemente fibras entre los adolescentes, se asoció ser mujer, tener mejor percepción de salud, y tener menos síntomas de hiperactividad-desatención; 2) tomar desayuno frecuentemente, se asoció a ser hombre y tener una menor frecuencia de problemas de conducta; 3) estirarse diariamente, se asoció a estar más conforme con apariencia física, tener mayor sentido de membresía escolar y buenas habilidades sociales; 4) hacer ejercicio vigoroso, 
Tabla 2. Características de los estudiantes y apoderados

\begin{tabular}{|c|c|c|}
\hline Estudiantes & $\mathbf{n}$ & $\%(95 \% \mathrm{Cl}) /$ Mean (DE) \\
\hline Edad & 560 & $11,5 \quad(1,19)$ \\
\hline Sexo (Mujeres) & 560 & $49,1 \quad(45,0-53,3)$ \\
\hline \multicolumn{3}{|l|}{ Curso } \\
\hline 5 Básico & 186 & $33,2(29,3-37,1)$ \\
\hline $6^{\circ}$ Básico & 186 & $33,2(29,3-37,1)$ \\
\hline $7^{\circ}$ Básico & 188 & $33,6(29,6-37,5)$ \\
\hline \multicolumn{3}{|l|}{ Percepción de Salud } \\
\hline Menos saludable que otros estudiantes & 94 & $16,9(13,8-20,0)$ \\
\hline Tan saludable como otros estudiantes & 281 & $50,4(46,3-54,6)$ \\
\hline Más saludable que otros estudiantes & 183 & $32,7 \quad(28,8-36,6)$ \\
\hline \multicolumn{3}{|l|}{ Conformidad con propio peso } \\
\hline No conforme con mi peso & 116 & $20,8(17,4-24,2)$ \\
\hline Más o menos conforme & 242 & $43,3(39,1-47,4)$ \\
\hline Muy conforme con mi peso & 200 & $35,9(31,9-40,0)$ \\
\hline \multicolumn{3}{|l|}{ Conformidad con apariencia física } \\
\hline No conforme con apariencia física & 83 & $14,9(11,9-17,9)$ \\
\hline Más o menos conforme & 218 & $39,1 \quad(35,1-43,2)$ \\
\hline Muy conforme con apariencia física & 257 & $46,0(41,8-50,1)$ \\
\hline \multicolumn{3}{|c|}{ Número de horas diarias viendo TV en un día de semana } \\
\hline$<1 \mathrm{~h}$ & 189 & $33,9(30,0-37,9)$ \\
\hline $1 \mathrm{~h}$ & 117 & $21,0(17,6-24,4)$ \\
\hline $2 \mathrm{~h}$ & 121 & $21,7(18,3-25,2)$ \\
\hline $3 \mathrm{~h}$ & 52 & $93 \quad(6,9-11,8)$ \\
\hline $4 \mathrm{~h}$ & 33 & $5,9 \quad(4,0-7,9)$ \\
\hline$\geq 5 \mathrm{~h}$ & 45 & $8,1 \quad(5,8-10,3)$ \\
\hline Membresía escolar & 548 & $3,7 \quad(0,7)$ \\
\hline SDQ-Síntomas emocionales & 555 & $4,5 \quad(2,3)$ \\
\hline SDQ-Problemas de conducta & 555 & $3,2 \quad(2,1)$ \\
\hline SDQ-Hiperactividad-desatención & 555 & $4,5 \quad(2,3)$ \\
\hline SDQ-Problemas con pares & 555 & $3,1 \quad(2,0)$ \\
\hline SDQ-Conducta pro-social & 555 & $7,4 \quad(1,9)$ \\
\hline \multicolumn{3}{|l|}{ Padres/apoderados } \\
\hline \multicolumn{3}{|l|}{ Estructura familiar } \\
\hline $0=$ Vive con uno de los padres & 223 & $40,0 \quad(36,0-44,0)$ \\
\hline $1=$ Vive con ambos padres & 334 & $60,0(55,9-64,0)$ \\
\hline \multicolumn{3}{|l|}{ Nivel educacional de apoderados } \\
\hline Básica incompleta & 71 & $12,9(10,1-15,2)$ \\
\hline Básica completa & 60 & $10,9 \quad(8,3-13,5)$ \\
\hline Secundaria incompleta & 88 & $16,0(13,0-19,1)$ \\
\hline Secundaria completa & 206 & $37,5(33,5-41,6)$ \\
\hline Superior-técnica incompleta & 22 & $4,0 \quad(2,4-5,7)$ \\
\hline Superior-técnica completa & 65 & $11,8 \quad(9,1-14,6)$ \\
\hline Universitaria incompleta & 12 & $2,2 \quad(1,0-3,4)$ \\
\hline Universitaria completa & 25 & $4,6 \quad(2,8-6,3)$ \\
\hline Salud Mental (GHQ-12) & 543 & $3,33 \quad(3,37)$ \\
\hline Funcionamiento familiar & 528 & $4,00 \quad(0,69)$ \\
\hline
\end{tabular}




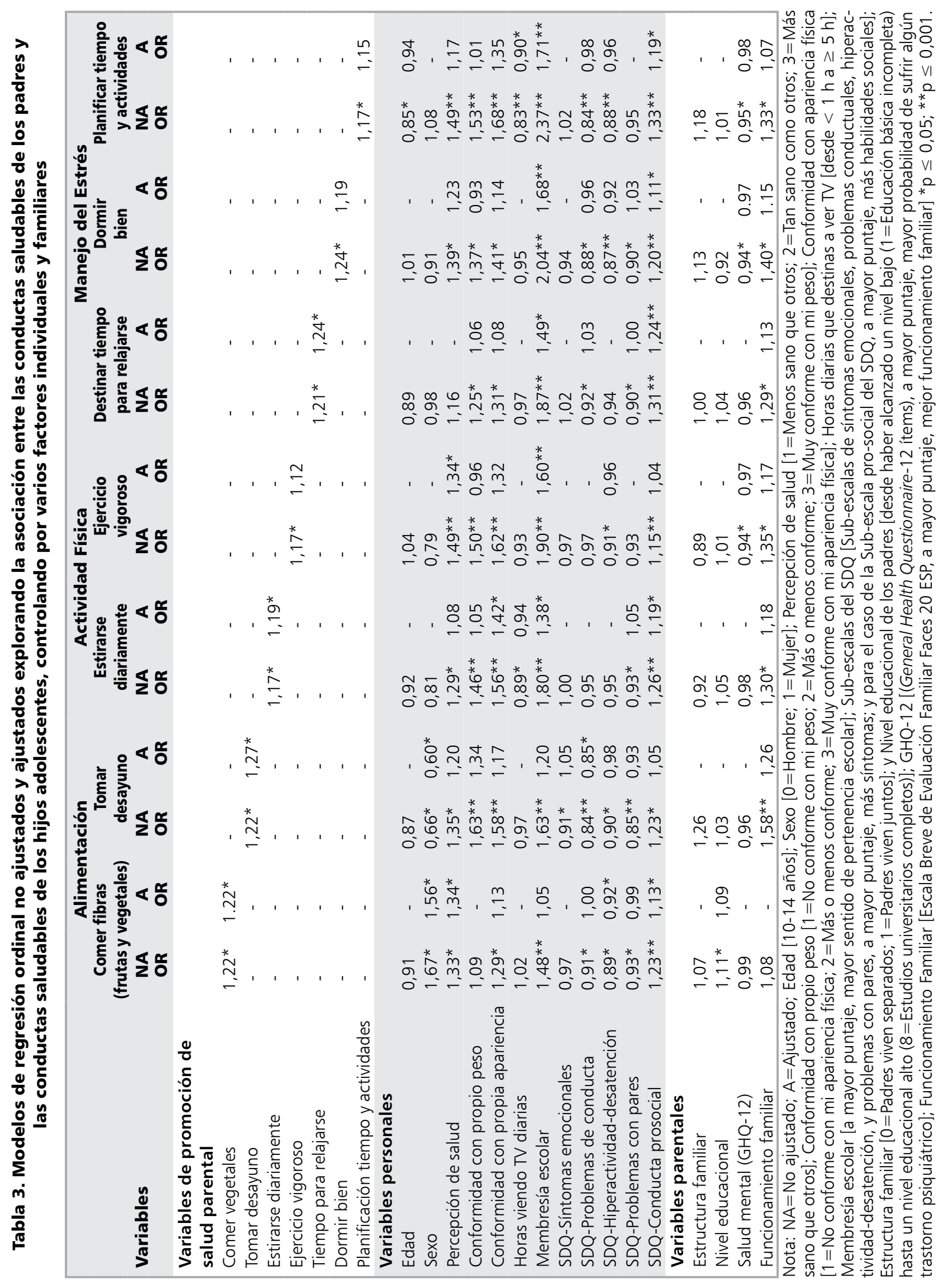


se asoció a tener una mejor percepción de salud y tener mayor sentido de membresía escolar. Las tres conductas de manejo del estrés: 5) destinar tiempo para relajarse; 6) dormir adecuadamente más días de la semana y 7) planificar tiempo y actividades, se asociaron a tener mayor sentido de membresía escolar y habilidades prosociales (Tabla 3 ).

\section{Discusión}

Este estudio entrega evidencia de la importancia que tienen algunas conductas promotoras de salud de los padres/apoderados en sus hijos adolescentes. Hasta el conocimiento de los autores, este es el primer estudio en Latinoamérica que explora esta asociación a través de la obtención de información independiente desde los adolescentes y de los padres.

Cierta explicación de estas asociaciones las podemos encontrar en la teoría de aprendizaje social, que propone que los procesos de imitación de las conductas de personas significativas están a la base del procesos de socialización de un estilo de vida saludable ${ }^{3,29}$. El Modelo Promotor de Salud $^{3}$ y el modelo socio-ecológico ${ }^{30-32}$ proponen que las conductas promotoras de salud estarían determinadas por la interacción de factores que se superponen en distintos niveles de influencia. A nivel interpersonal, se destaca la influencia de los padres ${ }^{19}$, a través de varias vías: i) transmisión de ciertas normas y expectativas ${ }^{33}$; ii) apoyo emocional de los padres ante el inicio o mantención de conductas saludables ${ }^{34,35}$; y iii) modelamiento de la conducta, a través del aprendizaje vicario ${ }^{36,37}$. Es posible que alguno de los mecanismos mencionados arriba también expliquen la asociación entre las habilidades sociales y las conductas saludables.

En relación a la influencia de otras variables, los indicadores de psicopatología de los adolescentes tuvieron distintos niveles de asociación. Por ejemplo, la presencia de síntomas emocionales no tuvo influencia, lo que podría ser considerado como un hallazgo, especialmente en relación a las conductas de manejo del estrés. En relación a tener problemas de conducta, encontramos que a mayor presencia de estos, menor probabilidad de tomar desayuno todos los días, lo que pudiera reflejar cierto oposicionismo ${ }^{38}$, pero también la asociación podría ser inversa. Por ejemplo, estudios iniciales en el efecto de la provisión de desayunos en los colegios (lo que aseguraría que lo niños lo reci- ban independiente de su condición económica) mostraban un efecto positivo en funcionamiento académico y en problemas conductuales en los co$\operatorname{legios}^{39,40}$; sin embargo, recientes ensayos clínicos aleatorios son menos auspiciosos en este sentido, especialmente con aquellos que deliberadamente se saltan el desayuno ${ }^{38,41}$. La presencia de síntomas de hiperactividad y desatención se asoció a menor probabilidad de comer fibras (frutas y vegetales), lo que ya ha sido encontrado en otros estudios, $y$ podría estar relacionado con el hecho que padres favorezcan, en estos niños, alimentos más ricos en carbohidratos, con menor consumo de vegetales y frutas ${ }^{42,43}$.

Algo especialmente interesante es lo que encontramos con el sentido de membresía escolar y la presencia de habilidades sociales, variables que se asociaron a varias conductas saludables, especialmente aquellas relacionadas con el manejo del estrés. Todo esto significa que ciertas conductas positivas se estén concentrando en un grupo de alumnos, reforzándose unas a otras. Nuevamente, no se puede asumir la dirección de la relación con este estudio, pero estas asociaciones deberán ser exploradas mejor, ya sea a través de estudios longitudinales o ensayos clínicos.

En relación a otras variables parentales, el estado de salud mental general, nivel educacional, $y$ funcionamiento o estructura familiar parecen tener un efecto menor en las conductas promotoras de salud exploradas. Esto no implica que haya otras conductas saludables, dentro de otros dominios como apoyo social, apreciación de la vida o responsabilidad en salud, no explorados en estos análisis, que puedan estar relacionados.

Entre las limitaciones, se debe mencionar en primer lugar que el diseño de análisis fue transversal, por lo que las asociaciones encontradas no son necesariamente causales. Por otro lado, los resultados deben ser considerados con cuidado antes de realizar generalizaciones, ya que se trata de una muestra reducida de estudiantes y padres/ apoderados de una ciudad del centro de Chile. Además, una proporción importante de apoderados contactados no quiso participar (34\%). En relación a esto último, hay cierta evidencia que muestra que el uso de un consentimiento activo, como en el presente estudio, podría haber hecho disminuir la participación de familias más vulnerables o de padres e hijos con conductas menos favorables con un desarrollo sano, como se ha 
evidenciado en otros estudios ${ }^{44,45}$; pero si este fuera el caso, nuestros resultados podrían tener como sesgo el subestimar las asociaciones y la magnitud del problema.

Estos hallazgos deben ser confirmados con otros diseños de investigación, como estudios longitudinales, donde se podrían determinar los efectos que tienen estas influencias parentales en el tiempo. También se deben proponer ensayos clínicos que permitan encontrar estrategias preventivas eficaces para mejorar las conductas saludables de los adolescentes y ayudar a mejorar el perfil epidemiológico de los adultos en Chile.

Agradecimientos: Agradecemos al personal de investigación que ha colaborado incansablemente en este estudio. Además, agradecemos la participación de los estudiantes y sus familias, así como a las autoridades de los establecimientos educacionales y del Departamento de Administración de Educación Municipal (DAEM) de la ciudad de San Felipe, Región de Valparaíso.

\section{Referencias}

1. Gore FM, Bloem PJN, Patton GC, Ferguson J, Joseph V, Coffey C, et al. Global burden of disease in young people aged 10-24 years: a systematic analysis. The Lancet 2011; 377 (9783): 2093-102.

2. Sawyer SM, Afifi RA, Bearinger LH, Blakemore SJ, Dick B, Ezeh AC, et al. Adolescence: a foundation for future health. Lancet 2012; 379 (9826): 1630-40.

3. Pender NJ, Murdaugh CL, Parsons MA. Health Promotion in Nursing Practice. 6th ed. New Jersey: Pearson; 2010.

4. Story M, Neumark-Sztainer D, French S. Individual and Environmental Influences on Adolescent Eating Behaviors. Journal of the American Dietetic Association 2002; 102 (3, Supplement): S40-S51.

5. Ali MM, Dwyer DS. Social network effects in alcohol consumption among adolescents. Addictive Behaviors 2010; 35 (4): 337-42.

6. Fletcher JM. Social interactions and smoking: Evidence using multiple student cohorts, instrumental variables, and school fixed effects. Health Economics 2010; 19 (4): 466-84.

7. van der Horst $\mathrm{K}$, Oenema A, Ferreira I, Wendel-Vos W, Giskes K, van Lenthe F, et al. A systematic review of environmental correlates of obesity-related dietary behaviors in youth. Health Education Research 2007; 22 (2): 203-26.
8. Burghardt J, Gordon A, Chapman N, Gleason P, Fraker T. The School Nutrition Dietary Assessment Study: school food service, meals offered and dietary intakes. Princeton, NJ: 1993.

9. Bursnall P. The relationship between physical activity and depressive symptoms in adolescents: a systematic review. Worldviews Evid Based Nurs 2014; 11 (6): 37682.

10. Kim HJ, Choi-Kwon S, Kim H, Park YH, Koh CK. Health-promoting lifestyle behaviors and psychological status among Arabs and Koreans in the United Arab Emirates. Res Nurs Health 2015; 38 (2): 133-41.

11. Sibold J, Edwards E, Murray-Close D, Hudziak JJ. Physical Activity, Sadness, and Suicidality in Bullied US Adolescents. Journal of the American Academy of Child \& Adolescent Psychiatry 2015; 54 (10): 808-15.

12. Fylkesnes K, Forde OH. The Tromso Study: predictors of self-evaluated health-has society adopted the expanded health concept? Soc Sci Med 1991; 32 (2): 141-6.

13. Vingilis ER, Wade TJ, Seeley JS. Predictors of adolescent self-rated health. Analysis of the National Population Health Survey. Canadian Journal of Public Health= Revue canadienne de santé publique 2002; 93 (3): 193-7.

14. Meland E, Haugland S, Breidablik HJ. Body image and perceived health in adolescence. Health Educ Res 2007; 22 (3): 342-50.

15. Mechanic D, Hansell S. Adolescent competence, psychological well-being, and self-assessed physical health. J Health Soc Behav 1987; 28 (4): 364-74.

16. Dyremyhr AE, Díaz E, Meland E. How adolescent subjective health and satisfaction with weight and body shape are related to participation in sports. J Environ Public Health 2014; 2014: 851932.

17. Gillison FB, Standage M, Skevington SM. Relationships among adolescents' weight perceptions, exercise goals, exercise motivation, quality of life and leisure-time exercise behaviour: a self-determination theory approach. Health Educ Res 2006; 21 (6): 836-47.

18. Chen MY, Lai LJ, Chen HC, Gaete J. Development and validation of the short-form Adolescent Health Promotion Scale. BMC Public Health 2014; 14: 1106.

19. Cruwys T, Bevelander KE, Hermans RCJ. Social modeling of eating: A review of when and why social influence affects food intake and choice. Appetite 2015; 86: 3-18.

20. Collins WA, Steinberg L. Adolescent development in interpersonal context. In: Darnon W, Eisemberg N, editors. Handbook of Child Psychology. 5th ed. New York, NY: Wiley; 2006. p. 1005-52. 
21. Collins WA, B L. Parent-adolescent relationships and influences. In: Lerner R, Steinberg L, editors. Handbook of Adolescent Psychology. New York, NY: Wiley; 2004. p. 331-61.

22. Chen MY, Wang EK, Yang RJ, Liou YM. Adolescent health promotion scale: development and psychometric testing. Public Health Nurs 2003; 20 (2): 104-10.

23. Walker SN, Sechrist KR, Pender NJ. The Health-Promoting Lifestyle Profile: development and psychometric characteristics. Nurs Res 1987; 36 (2): 76-81.

24. Walker SN, Kerr MJ, Pender NJ, Sechrist KR. A Spanish language version of the Health-Promoting Lifestyle Profile. Nurs Res 1990; 39 (5): 268-73.

25. Goodman A, Goodman R. Strengths and difficulties questionnaire as a dimensional measure of child mental health. J Am Acad Child Adolesc Psychiatry 2009; 48 (4): 400-3.

26. Araya R, Wynn R, Lewis G. Comparison of two self administered psychiatric questionnaires (GHQ-12 and SRQ-20) in primary care in Chile. Soc Psychiatry Psychiatr Epidemiol 1992; 27 (4): 168-73.

27. Zicavo N, Palma C, Garrido G. Adaptación y validación del Faces-20-ESP: Re-conociendo el funcionamiento familiar en Chillán, Chile. Revista Latinoamericana de Ciencias Sociales, Niñez y Juventud 2012; 10 (1): 219-34.

28. World Medical A. World medical association declaration of Helsinki: Ethical principles for medical research involving human subjects. JAMA 2013; 310 (20): 21914.

29. Blair JE. Social learning theory: strategies for health promotion. AAOHN J 1993; 41 (5): 245-9.

30. Story M, Kaphingst KM, Robinson-O'Brien R, Glanz K. Creating Healthy Food and Eating Environments: Policy and Environmental Approaches. Annual Review of Public Health 2008; 29 (1): 253-72.

31. Diallo FB, Potvin L, Bedard J, Larose F. [Participation of parents in a nutritional education program in schools and development of eating behaviours of children]. Canadian Journal of Public Health = Revue canadienne de santé publique 2014; 105 (6): e425-30.

32. Bronfenbrenner U. Ecological systems theory. Six theories of child development: Revised formulations and current issues. London, England: Jessica Kingsley Publishers; 1992. p. 187-249.

33. Fisher L, Chesla CA, Skaff MM, Gilliss C, Mullan JT, Bartz RJ, et al. The family and disease management in Hispanic and European-American patients with type 2 diabetes. Diabetes Care 2000; 23 (3): 267-72.

34. Neumark-Sztainer D, Wall M, Perry C, Story M. Correlates of fruit and vegetable intake among adolescents:
Findings from Project EAT. Preventive Medicine 2003; 37 (3): 198-208.

35. Morrissey JL, Janz KF, Letuchy EM, Francis SL, Levy SM. The effect of family and friend support on physical activity through adolescence: a longitudinal study. The International Journal of Behavioral Nutrition and Physical activity 2015; 12 (1): 103.

36. Ritchie LD, Welk G, Styne D, Gerstein DE, Crawford PB. Family environment and pediatric overweight: What is a parent to do? Journal of the American Dietetic Association 2005; 105 (5): S70-S9.

37. Cooke LJ, Wardle J, Gibson E, Sapochnik M, Sheiham A, Lawson M. Demographic, familial and trait predictors of fruit and vegetable consumption by pre-school children. Public Health Nutrition 2004; 7 (02): 295-302.

38. Murphy S, Moore G, Tapper K, Lynch R, Clarke R, Raisanen $\mathrm{L}$, et al. Free healthy breakfasts in primary schools: a cluster randomised controlled trial of a policy intervention in Wales, UK. Public Health Nutrition 2011; 14 (02): 219-26.

39. Murphy J, Pagano ME, Nachmani J, Sperling P, Kane $S$, Kleinman RE. The relationship of school breakfast to psychosocial and academic functioning: Cross-sectional and longitudinal observations in an inner-city school sample. Archives of Pediatrics \& Adolescent Medicine 1998; 152 (9): 899-907.

40. Bro RT, Shank L, Williams R, McLaughlin TF. The Effects of an In-Class Breakfast Program on Attendance and On-Task Behavior of High School Students. Child \& Family Behavior Therapy 1994; 16 (3): 1-8.

41. Adolphus K, Lawton CL, Dye L. The effects of breakfast on behavior and academic performance in children and adolescents. Frontiers in Human Neuroscience 2013; 7: 425.

42. Froehlich TE, Anixt JS, Loe IM, Chirdkiatgumchai V, Kuan L, Gilman RC. Update on Environmental Risk Factors for Attention-Deficit/Hyperactivity Disorder. Current Psychiatry Reports 2011; 13 (5): 333-44.

43. Howard AL, Robinson M, Smith GJ, Ambrosini GL, Piek JP, Oddy WH. ADHD Is Associated With a "Western" Dietary Pattern in Adolescents. Journal of Attention Disorders 2011; 15 (5): 403-11.

44. Frissell KC, McCarthy DM, D Amico EJ, Metrik J, Ellingstad TP, Brown SA. Impact of Consent Procedures on Reported Levels of Adolescent Alcohol Use. Psychology of Addictive Behaviors 2004; 18 (4): 307-15.

45. Chartier M, Stoep AV, McCauley E, Herting JR, Tracy M, Lymp J. Passive Versus Active Parental Consent: Implications for the Ability of School-based Depression Screening to Reach Youth at Risk. The Journal of school health 2008; 78 (3): 157-86. 